\title{
The stenosed heart revived by homoeopathy
}

\begin{abstract}
In mitral valve disease, the mitral valve, which is located between your left heart chambers (left atrium and left ventricle), doesn't work properly.

Types of mitral valve disease include:

Mitral valve regurgitation: In this condition, the flaps (leaflets) of the mitral valve don't close tightly, causing blood to leak backward into the left atrium of your heart. If not treated, it can result in heart muscle damage. The most common cause of blood leakage is mitral valve prolapse, in which the leaflets bulge back into the left atrium as your heart contracts.

Mitral valve stenosis: In this condition, the flaps of the mitral valve become thick or stiff, and they may fuse together. This results in a narrowed valve opening and reduced blood flow from the left atrium to the left ventricle.

Homeopathy is one of the most popular holistic systems of medicine. The selection of remedy is based upon the theory of individualization and symptoms similarity by using holistic approach. This is the only way through which a state of complete health can be regained by removing all the sign and symptoms from which the patient is suffering. The aim of homeopathy is not only to treat mitral valve disease but to address its underlying cause and individual susceptibility. As far as therapeutic medication is concerned, several remedies are available to treat mitral valve disease that can be selected on the basis of cause, sensations and modalities of the complaints. For individualized remedy selection and treatment, the patient should consult a qualified homeopathic doctor in person.
\end{abstract}

Keywords: mitral valve regurgitation, mitral valve stenosis, individualization, susceptibility

\author{
Volume 2 Issue 5 - 2017
}

\section{Parth Aphale}

DY Patil Homoeopathic Medical College \& Research Centre, India

Correspondence: Parth Aphale, DY Patil Homoeopathic Medical College \& Research Centre, Pune, India, Email parth.aphale@gmail.com

Received: February 24, 2017 | Published: April 10, 2017

\section{Introduction}

A male patient, XYZ, 65YRS. old came to my clinic on 30/08/2015 complaining of breathlessness, heaviness of chest and chest pain. Dyspnoea <exertion, lying down > sitting erect. Constricting Pain in left side of chest radiating down the left hand with heaviness++. Feeling of suffocation. Palpitations ++ . K/C/O-HTN.

\section{Chief complaint}

Chest pain with heaviness, dyspnea on exertion.

\section{Associated complaint}

No associated complaint.

\section{Past history}

Angioplasty 4yrs ago.

\section{Family history}

No major illness in the family (Table 1) (Table 2).

\section{Systemic examination}

CVS: S1, S2 heard, Mid Diastolic murmur heard.

2-D Echo- Mild Mitral Regurgitation.

Trivial Aortic regurgitation.

Mild Concenteric Ventricular Hypertrophy.
LVEF- 25\%.

No Thrombus or Pericardial Effusion.

Dilated L.A.

Table I Personal history

\begin{tabular}{ll}
\hline Appetite & Normal \\
\hline Thirst & Increased for large quantities of water \\
Cravings & Sweet ++ \\
Aversions & Nothing Specific \\
Bowels & Hard stools with straining \\
Urine & Normal \\
Perspiration & Scanty, no staining, no odor \\
Thermals & Hot(wants fan++, < hot weather, >cold) \\
Sleep & Normal \\
Dreams & Nothing specific \\
\hline
\end{tabular}

\section{Clinical diagnosis}

Mitral \& trivial aortic regurgitation.

\section{Acute totality}

i. Constricting pain in left side of chest radiating down the left hand with heaviness ++ . 
ii. Dyspnoea $<$ exertion, lying down $>$ sitting erect.

iii. Heaviness of chest <exertion, lying down.

iv. Suffocation, Breathlessness.

v. Palpitations.

vi. Pulse intermittent.

Table 2 General examination

\begin{tabular}{ll}
\hline General examination & Vital data \\
\hline Lean, thin, tall & Temperature- Afebrile \\
Dark complexion & Pulse- $60 / \mathrm{min}$, intermittent \\
& B.P- $150 / 100 \mathrm{~mm} . \mathrm{Hg}$. \\
& R.R- $18 / \mathrm{min}$. \\
\hline
\end{tabular}

\section{Repertorial totality}

a) CHEST - PAIN - constricting.

b) CHEST - PALPITATION of heart - exertion.

c) CHEST - OPPRESSION - lying, while.

d) GENERALS - PULSE - intermittent.

e) CHEST-MURMURS-CARDIAC MURMURS

f) CHEST-MURMURS-CARDIAC MURMURS-VALVULAR-MITRAL VALVE (Figure 1).

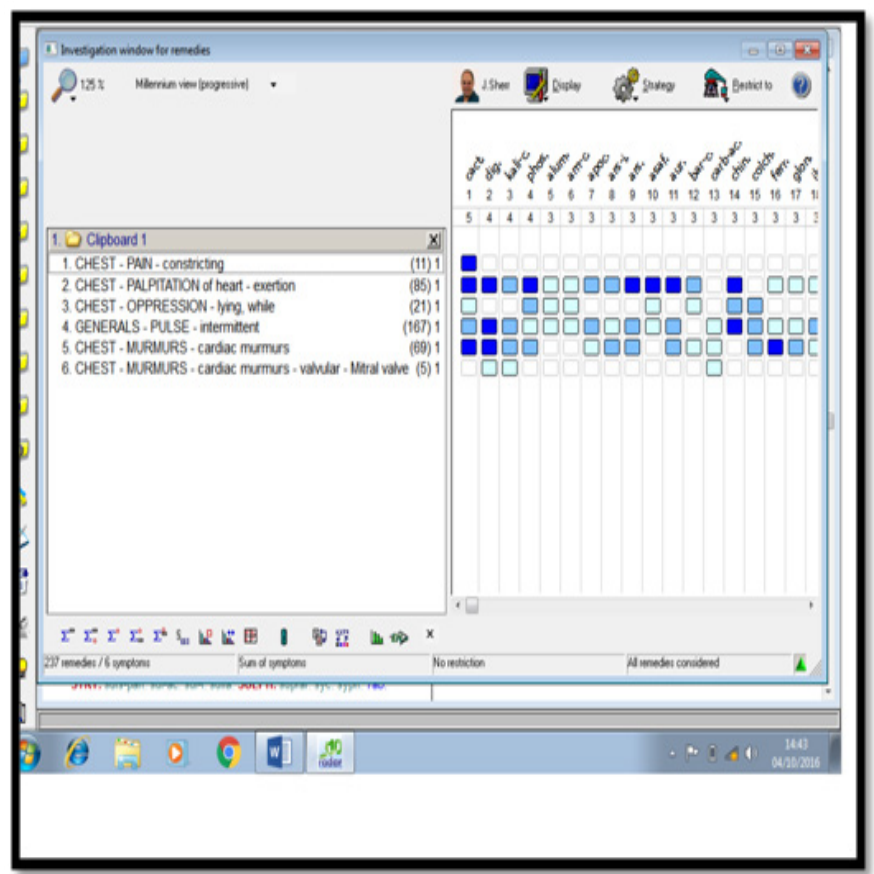

Figure I Repertorisation.

\section{Probable remedies}

Cactus Grandiflorus, Digitalis, Kalmia Latifolia

\section{Indicated remedy}

$6 / 12 / 2016$

\section{Prescription}

Digitalis 200, 3 pills T.D.S, x7days (Table 3) (Figure 2) (Figure 3).

Table 3 Follow up chart

\begin{tabular}{|c|c|c|}
\hline Follow up & Symptoms & Remedy \\
\hline $7 / 9 / 2015$ & $\begin{array}{l}\text { Slight Improvement. } \\
\text { Pain and heaviness } \\
\text { in chest slightly less. } \\
\text { Breathlessness on } \\
\text { exertion+, Palpitations+ }\end{array}$ & $\begin{array}{l}\text { Digitalis } 200 \text { T.D.S for } \\
\text { I5days. }\end{array}$ \\
\hline $26 / 09 / 2015$ & $\begin{array}{l}\text { Better. Pain and heaviness } \\
\text { much less. Breathlessness } \\
\text { much better, palpitations } \\
\text { much less }\end{array}$ & $\begin{array}{l}\text { Digitalis } 200 \text { T.D.S for } \\
\text { I } 5 \text { days. }\end{array}$ \\
\hline $15 / 10 / 2015$ & $\begin{array}{l}\text { Much better. Occasional } \\
\text { pain, No heaviness, } \\
\text { breathlessness only } \\
\text { on over-exertion, no } \\
\text { palpitations }\end{array}$ & $\begin{array}{l}\text { Digitalis } 200 \text { T.D.S for } \\
\text { Imonth }\end{array}$ \\
\hline $20 / 11 / 2015$ & $\begin{array}{l}\text { Much better. No Pain, } \\
\text { No heaviness, occasional } \\
\text { breathlessness, no } \\
\text { palpitations }\end{array}$ & $\begin{array}{l}\text { Digitalis } 200 \text { T.D.S for } \\
\text { Imonth }\end{array}$ \\
\hline $25 / 12 / 2015$ & $\begin{array}{l}\text { Much better No } \\
\text { Complaints }\end{array}$ & $\begin{array}{l}\text { Digitalis } 200 \text { T.D.S for } \\
\text { Imonth }\end{array}$ \\
\hline $30 / 01 / 2016$ & No complaints & $\begin{array}{l}\text { Digitalis } 200 \text { T.D.S for } \\
\text { Imonth }\end{array}$ \\
\hline $3 / 2 / 2016$ & Breathless+, Palpitations+ & $\begin{array}{l}\text { Digitalis } 200 \text { T.D.S for } \\
\text { Imonth }\end{array}$ \\
\hline $5 / 3 / 2016$ & Better & $\begin{array}{l}\text { Digitalis } 200 \text { T.D.S for } \\
\text { Imonth }\end{array}$ \\
\hline $2 / 4 / 2016$ & $\begin{array}{l}\text { Slight Breathlessness } \\
\mathrm{H} / \mathrm{O} \text { - Overexertion. }\end{array}$ & $\begin{array}{l}\text { Digitalis } 200 \text { T.D.S for } \\
\text { Imonth }\end{array}$ \\
\hline $10 / 5 / 2016$ & $\begin{array}{l}\text { Much better. No } \\
\text { Breathlessness }\end{array}$ & $\begin{array}{l}\text { Digitalis } 200 \text { T.D.S for } \\
\text { Imonth }\end{array}$ \\
\hline $9 / 6 / 2016$ & $\begin{array}{l}\text { No Complaints. Advised } \\
\text { 2-D Echo on next follow } \\
\text { up }\end{array}$ & $\begin{array}{l}\text { Digitalis } 200 \text { T.D.S for } \\
\text { Imonth }\end{array}$ \\
\hline $15-07-2016$ & $\begin{array}{l}\text { 2-D ECHO shows } \\
\text { normal L.V size, and } \\
\text { resolved mitral stenosis }\end{array}$ & S.LT.D.S for I month \\
\hline $18-08-2016$ & $\begin{array}{l}\text { No Complaints. Much } \\
\text { Better }\end{array}$ & $\begin{array}{l}\text { S.L T.D.S for I month. } \\
\text { The dose of Allopathic } \\
\text { medicines has been } \\
\text { reduced. Anti-Hypertension } \\
\text { medicine has been reduced } \\
\text { form } 3 \text { tablets per day to } \\
\text { only } 1 / 2 \text { tablet at night as } \\
\text { B.P is constantly normal. } \\
\text { Tab. Ecosprin is going on } \\
\text { only now. }\end{array}$ \\
\hline $6 / 12 / 2016$ & $\begin{array}{l}\text { No Complaints. Medicnes } \\
\text { have reduced. Patient is } \\
\text { happy. Has done ECG. It } \\
\text { is perfectly normal. No } \\
\text { new changes in ECG. }\end{array}$ & $\begin{array}{l}\text { Stop treatment. Follow up } \\
\text { as and when needed. }\end{array}$ \\
\hline
\end{tabular}
is perfectly normal. No anges in ECG.

Digitalis. 
Post treatment 2-D Echo: Normal L.V. Size, LVEF-36\%.

LA, RA, RV Normal.

Mitral leaflets normal texture and excursion.

No Mitral Regurge.

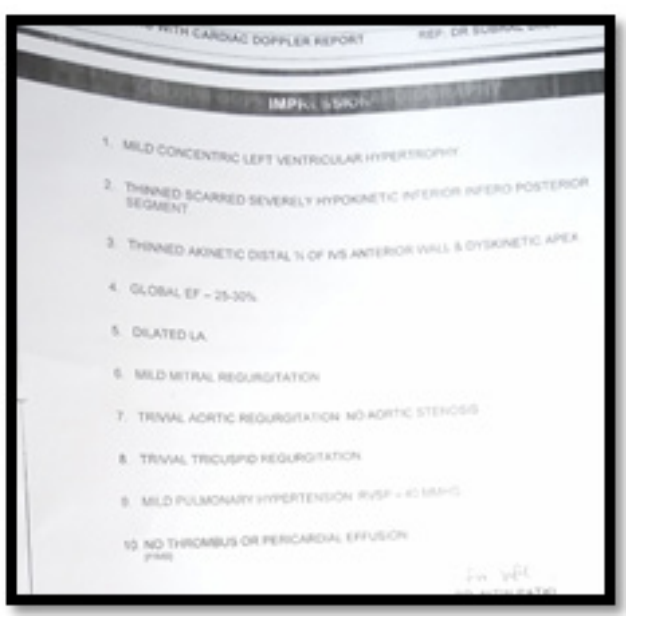

Figure 2 Before treatment.

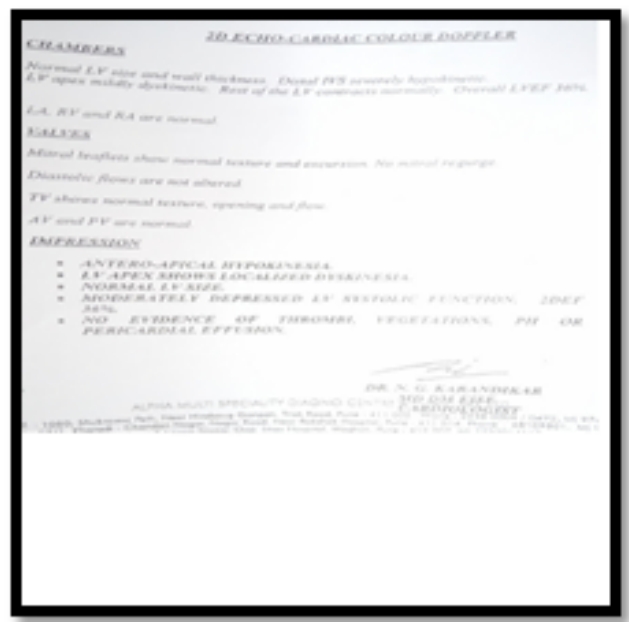

Figure 3 After treatment.

\section{Case discussion}

From the above case, it is evident that Digitalis proved to be the similimum. If we were to compare the reports of 2-D Echo before and after treatment, then before starting the treatment, there was mild L.V Hypertrophy, effective volume was $25-30 \%$, dilated L.A,mitral stenosis, aortic regurgitation, pulmonary hypertension. After 10 months of treatment, no mitral stenosis as well as regurgitation, effective volume increased from $25 \%$ to $36 \%$, L.A normal, mitral leaflets show normal texture and excursion, Normal L.V size no pulmonary hypertension. This shows that Digitalis has potent action on the heart, mainly the Mitral valves, and left ventricle. If we look in to the Clinical Materia Medica of Digitalis, it is one of the fore-runners for mitral valve disease, mainly mitral stenosis. If we see at some of the rubrics that were considered in this case, GENERALS-PULSEintermittent, CHEST- MURMURS- CARDIAC MURMURS,CHESTMURMURS- CARDIAC MURMURS- VALVULAR- MITRAL VALVE, we see that all these are pathological generals. Thus pathological generals are very much important while treating such deep seated diseases.

\section{Conclusion}

To conclude, deep knowledge of Materia Medica along with pathological generals and their practical application is very much important in treating deep seated organic chronic disease.

\section{Acknowledgements}

None.

\section{Conflict of interest}

Author declares that there is no conflict of interest. 